\title{
¿"SUTILEZAS" DE LA DISCRIMINACIÓN Y LA VIOLENCIA BASADA EN EL GÉNERO? SITUACIÓN DE LOS Y LAS ESTUDIANTES DE PREGRADO Y POSGRADO DE UNA FACULTAD DE MEDICINA EN BOGOTA D.C.
}

\author{
SANDRA PATRICIA MORENO REALPHE ${ }^{1,}$ PABLO ALFONSO SANABRIA FERRAND², LUIS ARTEMO \\ GONZÁLEZZ, QUEVEDO CLAUDIA LUCÍA VALENCIA CEDEÑO". \\ ${ }^{1}$ Médica, Magister en Estudios de Familia, Docente Investigadora, Grupo Salud y Comportamiento Facultad de \\ Medicina, Universidad Militar Nueva Granada. \\ ${ }^{2}$ Psicólogo, Epidemiólogo, Magister en Psicología con énfasis en Salud, Docente Investigador, Grupo Salud y \\ Comportamiento, Facultad de Medicina, Universidad Militar Nueva Granada. \\ ${ }^{3}$ Psicólogo, Epidemiólogo, Magister en Psicología con énfasis en Salud, Docente Investigador, Grupo Salud y \\ Comportamiento, Facultad de Medicina, Universidad Militar Nueva Granada. \\ ${ }^{4}$ Psicóloga, Especialista en Psicología Clínica, Magister en Psicología Comunitaria, Asistente de investigación, Grupo \\ Salud y Comportamiento, Facultad de Medicina, Universidad Militar Nueva Granada. \\ Correspondencia: sandra.moreno@unimilitar.edu.co \\ Dirección Postal: Transversal 3 \# 49-00 Facultad de Medicina. Bogotá, Colombia
}

Recibido: 30 Abril 2015 Aceptado: 16 Junio 2015

\begin{abstract}
Resumen
Introducción: La Violencia basada en el Género (VBG) es un problema complejo, multifactorial y sus efectos generan riesgos y problemas para la salud reproductiva, enfermedades crónicas, consecuencias psicológicas, lesiones y muerte. El objetivo fue caracterizar la violencia basada en el género (VBG) en una Facultad de Medicina en Bogotá D.C., la cual ya ha sido comprobada en Facultades de Medicina de otros países como Japón y Canadá.

Materiales y métodos: Estudio exploratorio caracterizando la VBG en la Facultad de Medicina de la Universidad Militar Nueva Granada (UMNG). La población estuvo conformada por estudiantes de pregrado cursando semestres clínicos y estudiantes de posgrado. El muestreo intencionado con estudiantes que voluntariamente accedieran participar por semestre. 201 estudiantes (170 pregrado y 31 posgrados), respondieron un cuestionario, entre septiembre de 2012 y febrero de 2013. Se identificó el tipo de VBG percibida, su frecuencia, la respuesta secundaria al suceso y sus consecuencias.

Resultados: La mayoría de los/las estudiantes percibieron este tipo de violencia asociada con el hecho de ser mujer (94\%), seguido por la orientación no heterosexual en un $8.5 \%$, las creencias religiosas (5.1\%) y la raza (3.4\%). El tipo de violencia más frecuente fue la psicológica $65.7 \%$, la sexual en un $35.3 \%$ y física en un $6.5 \%$. Se observó que la respuesta más frecuente fue ignorar la situación (41\%), seguida de contarle a un amigo (26.8\%). Las consecuencias identificadas fueron ignorar lo sucedido y actuar como si este hecho no se hubiera presentado, seguida por la decisión de aumentar su esfuerzo en la materia en mención. Conclusiones: La VBG en este contexto es una problemática presente y que dista de ser un hecho aislado o sutil. Lo que reafirma los planteamientos de la Convención sobre la eliminación de todas las formas de discriminación contra la mujer - CEDAW (Convention on elimination of all forms of discrimination against women) y evidencia la importancia
\end{abstract}


de analizar las condiciones de la violencia contra la mujer en los diferentes ámbitos. El comportamiento de la VBG en la población estudiada, tuvo una distribución similar a la encontrada en los estudios desarrollados por otros autores. De allí que sea necesario reflexionar que en un entorno de salud - donde se manejan conceptos como bienestar, equidad, diversidad, respeto a los pacientes se podría estar manejando un lenguaje paralelo y contradictorio. Es importante analizar si la exigencia académica y éxito, se pueden ver erróneamente asociados con técnicas restrictivas del aprendizaje, lo que podría explicar por qué una de las consecuencias más enunciadas por los estudiantes fue que la situación los llevó a esforzarse más en su cotidianidad. Lo anterior podría llevar a un círculo vicioso de reconocimiento y validación que puede generar una gran limitante para la formulación de estrategias de cambio a futuro.

Palabras clave: Violencia, género, acoso sexual, educación médica.

\title{
SUBTLETIES OF DISCRIMINATION AND VIOLENCE BASED ON GENDER? SITUATION OF COURSES OF GRADE AND POSTGRADE STUDENTS OF A MEDICINE FACULTY EN BOGOTÁ D.C.
}

\begin{abstract}
Introduction: Gender based violence (GBV) include all types of Human Rights violations due to the sex, age, race, ethnicity, religious beliefs and sexual orientation. GBV is a complex problem and its effects generate risks and reproductive health problems, chronic illness, psychological consequences, injuries even death.

Materials and Methods: Exploratory design for the characterization of the GBV at the Faculty of Medicine of the Military University in Bogotá. The universe were premed students and residents, the sampling was by convenience with students that agreed to participate in the study. 201 students, 170 of premed and 31 residents, between September 2012 and February 2013, answered the survey. The survey identified the type, frequency, the response and the consequences of the GBV at the medical school.

Results: Most of the GBV were due to being a woman (94\%), followed by sexual orientation (8.5\%), religious beliefs $5.1 \%$ and race (3.4\%). The psychological violence was the most frequently perceived $65.7 \%$, sexual violence was present on $35.3 \%$ and physical violence on a $6.5 \%$. The most used response was ignoring the situation (41\%), followed by telling a friend (26.8\%). The consequences identified were denial and the decision of work harder at the hospital.

Discussion: As a finding, the GBV in this context is present and is not an isolated or subtle condition. This reaffirms CEDAW's (Convention on elimination of all forms of discrimination against women) approaches related with violence against women as the main type of violence that requires attention. The behavior of GBV at the Faculty of Medicine at the Military University was similar with findings on related studies. Therefore is necessary to make a reflection about why, if on medical education context are used concepts like wellbeing, equity, diversity, respect, it could be a parallel and contradictory language (hidden curricula). Also it is important to analyze exists a miss relation between academic success with restrictive and punitive educational techniques due to cultural beliefs.
\end{abstract}

Keywords: Violence, Gender, Medical Education, Sexual Harassment. 


\title{
¿SUTILEZAS DA DISCRIMINAÇÃO E A VIOLÊNCIA BASADA NO GÉNERO? SITUAÇÃO DUMA FACULDADE DE MEDICINA NA BOGOTÁ D.C.
}

\author{
Resumo
}

Introdução: A Violência baseada no Género (VBG) é um problema complexo, multifatorial y seus efeitos geram riscos e problemas para a saúde reprodutiva, doenças crónicas, consequências psicológicas, lesiones e morte. O objetivo foi caracterizar a violência baseado no género (VBG) em uma faculdade de Medicina em Bogotá D.C. como já foi feito em faculdades de medicina de países como Japão e Canadá.

Materiais e métodos: Estudo de exploração que caracteriza a VBG na Faculdade de Medicina da Universidade Militar Nueva Granada (UMNG). A população foi formada por estudantes de cursos de graduação que fazem semestres cínicos e estudantes de pós-graduação. O amostragem foi com estudantes voluntários por semestre. 201 estudantes (170 de cursos de graduação e 31 de pósgraduação) responderam um questionário, entre setembro de 2012 e fevereiro de 2013. Identificouse o tipo de VBG percebida, a frequência, a resposta secundaria ao feito e as consequências.

Resultados: a maioria dos (as) estudantes perceberam este tipo de violência associada com o feito de ser mulher (94\%), seguido pela orientação no heterossexual num 8.5\%; as crenças religiosas (5.1\%) e a raça (3.4\%). O tipo de violência mais frequente foi a psicológica 65.7\%, a sexual num 35.3\% e física num 6.5\%. Observou-se que a reposta más frequente foi ignorar a situação (41\%), seguida de falar com um amigo (26.8\%). Ignorar o que aconteceu e atuar como si o feito não presentou-se foram as consequências identificadas, seguido pela decisão de aumentar o esforço na matéria em menção.

Conclusões: A VBG neste contesto é uma problemática presente, que no é um feito asilado ou sutil. Esso reafirma as afirmações da Convenção sobre a eliminação de todas as formas de discriminação contra a mulher - CEDAW (Convention on elimination of all forms of discrimination against women) e evidencia a importância de analisar as condições da violência contra a mulher nos diferentes contextos. O comportamento da VBG na população estudada, teve uma distribuição similar ao que acharam nos estudos desenvolvidos por outros autores. É preciso reflexionar que é um entorno de saúde - donde manejam-se conceitos como bem-estar, equidade, diversidade, respeito aos pacientes - pode-se falar um linguaje paralelo e contraditório. É importante analisar se a exigência académica e sucesso percebem-se erradamente em associação com técnicas restritivas de aprendizagem, o que pode explicar por que uma das consequências mais enunciadas pelos estudantes foi que a situação levo-os no sentido dum esforço mais em sua cotidianidade. $\mathrm{O}$ anterior pode levar a um circulo vicioso de reconhecimento e validação que pode gerar uma grande limitante para a formulação de estratégias de cambio ao futuro.

Palavras-chave: violência, género, acoiço sexual, educação médica. 


\section{Introducción}

La violencia en cualquiera de sus formas es una vulneración de las garantías legales, de los mínimos éticos que protegen la libertad y la dignidad de todos los seres humanos sin distinción (de sexo, edad, etnia, estrato, entre otros) en ámbitos civiles, políticos, económicos, sociales y culturales, tanto en lo público como en lo privado (Derechos Humanos).

Por lo anterior, el analizar las condiciones de la violencia desde el Enfoque de Derechos es prioritario. Ya que este marco conceptual integra estándares y principios, y le apuesta al desarrollo de las capacidades de los/las titulares de derechos para la realización de los Derechos Humanos (DDHH). Este enfoque representa una forma de ocuparse del impacto de las desigualdades de poder y un instrumento para la transformación del conflicto.

El vínculo entre el Enfoque de derechos y Género radica en el sujeto como el titular de derechos humanos. Este sujeto requiere el desarrollo de capacidades para ejercer sus derechos y superar las desventajas y la exclusión a partir de las diferencias (1).

Cuando las sociedades, instituciones o las personas, realizan cualquier acto de distinción, exclusión o restricción hacia otras, basados en su sexo, edad, raza, etnia, creencia y orientación sexual; se incurre en discriminación. La discriminación es por sí misma una forma de interacción violenta.

Es por ello que desde su concepción más amplia, la Violencia basada en el Género (VBG) incluía todo tipo de vulneración de los Derechos Humanos con ocasión de su sexo, edad, raza, etnia, creencia y orientación sexual. En la actualidad, la definición se encuentra más vinculada al concepto de violencia contra la mujer. Lo anterior debido a que en los diversos estudios realizados, en las estadísticas a nivel mundial, es la mujer por su condición, la más afectada por la discriminación $(2,3)$.

Por ello en el artículo No. 1 de la Convención sobre la Eliminación de todas las formas de discriminación contra la mujer CEDAW (4), se estableció discriminación como:

"Toda distinción, exclusión o restricción, basada en el sexo que tenga por objeto o por resultado anular el reconocimiento, goce o ejercicio por la mujer de los derechos humanos y las libertades fundamentales en la esfera política, económica, social, cultural y civil o en cualquier otra esfera".

Y así mismo se definió la VBG como:
"Todo acto de violencia basada en el género que tiene como resultado posible o real, un daño físico, sexual o psicológico, incluidas las amenazas, la coerción o la privación arbitraria de la libertad, ya sea que ocurra en la vida pública o en la vida privada"

La Violencia de Género se da en todos los ámbitos y por parte de diversos agresores, desde la pareja y familiares, hasta desconocidos (5-7). Constituye un fenómeno extendido con características y matices diferentes. Lo anterior pudo observarse en el estudio realizado por el Instituto Nacional de Medicina Legal y Ciencias Forenses, Masatugó, donde se recopilaron los datos 2004-2008 de lesiones de causa externa y se realizó un análisis exhaustivo de las condiciones de las mujeres. En este contexto, se identificó a la violencia contra la mujer, como "producto de la perpetuación de formas de dominación y de los patrones patriarcales entre géneros". De igual manera evidenció que en la medida en que las mujeres incursionan de manera más amplia en los distintos escenarios de socialización, las actitudes y prácticas discriminatorias y violentas se pueden observar en otras instancias del desempeño social (8-10).

En ese sentido, los ámbitos universitarios a los cuales las mujeres han incursionado de forma creciente en los últimos años, se configuran en espacios donde podría reproducirse la violencia $(11,12)$. En el caso del presente estudio se identificó la violencia en la facultad de medicina de la Universidad Militar y sus sitios de práctica(13).

La VBG se ha identificado como un problema complejo, multifactorial, influido por factores sociales, económicos, psicológicos, jurídicos, culturales y biológicos (14). Los efectos de este tipo de violencia generan riesgos $y$ problemas para la salud reproductiva, enfermedades crónicas, consecuencias psicológicas, lesiones y la muerte (15).

\section{La Violencia basada en el género en los programas de formación médica}

Las Violencias en el ámbito educativo han sido estudiadas por diferentes instituciones en Colombia. Es el caso de la Universidad de Caldas, donde se encontró que estas formas de discriminación y violencia basada en el género se presentan en todas las facultades y no sólo afectan a estudiantes sino también a administrativos y docentes. En cuanto a la discriminación y la violencia basada en el género hacia los/las estudiantes, se encontró que la Facultad de Medicina es la que presenta los porcentajes más altos (16).

Específicamente la Violencia contra las y los estudiantes en los programas de pregrado y posgrado de medicina ha sido estudiada por diversos autores $(12,17,18)$, planteando que 
los médicos y médicas son susceptibles de experimentar y así mismo perpetrar, abuso y discriminación. La Universidad de Mc Master en Canadá (19), realizó en el año 1996, un estudio acerca de la prevalencia de la violencia en estudiantes de medicina. Específicamente para la violencia contra la mujer se encontró una prevalencia del 71\%, para la violencia secundaria a su orientación sexual se presentó en un $10.2 \%$, para acoso sexual se reportó en un $92.2 \%$ y para violencia física, un hallazgo no esperado por los investigadores, estuvo en el 54\%. Este estudio propuso iniciativas educativas, de comportamiento y estructurales para incorporar en los programas de formación médica y erradicar estas prácticas.

Otros estudios han relacionado la VBG con la aplicación de la escala de Burnout de Maslach y el inventario de Leymman de terror psicológico en los estudiantes de posgrados médicos (20), encontrando que estas condiciones se presentaron de igual manera en hombres que en mujeres pero que hubo un mayor grado de despersonalización en mujeres que en hombres.

En 2003, se realizó un estudio de prevalencia de VBG en estudiantes de medicina de 6 facultades en Japón (21), encontrando que el 68.5\% de los participantes refirió algún tipo de violencia. Para el caso de VBG, por su condición de ser mujer, se presentó el acoso sexual en el 51.4\% de las estudiantes. No se exploró la violencia por creencias, orientación sexual o raza.

En el año 2011 la Asociación Médica Americana (22) realizó una junta cuyo objetivo principal fue analizar estrategias para la optimización del ambiente de aprendizaje, y profundizó acerca de la temática violencia (misstreatment). Esta Junta definió maltrato así:

"Cuando de manera intencional o no, el comportamiento muestra falta de respeto por la dignidad de otros e interfiere sin razón con el proceso de aprendizaje. Los ejemplos de maltrato incluyen acoso sexual, discriminación o acoso basados en raza, religión, etnia, género u orientación sexual, el castigo físico o psicológico y el uso de evaluación y otras formas de asesoría de una manera punitiva".

La Junta presentó resultados del año 2010 y 2011, con una prevalencia entre el 11 y el $40 \%$ concordantes con estudios presentados anteriormente.

\section{La mujer en la carrera de medicina}

Es claro que desde la revolución de los años 70 las mujeres han empezado a ocupar espacios en los procesos de formación médica, es innegable que en la actualidad superan en número a los hombres en los programas de pregrado. Sin embargo, Newman (23) afirmó que persiste el concepto de techo de cristal en la jerarquía médica, impidiendo que estas puedan ascender en ciertas especialidades. Así como las dificultades derivadas del cuidado de los hijos y del hogar que todavía son atribuidas en gran parte al rol femenino, pueden interferir en las percepciones que tienen los profesores y la comunidad con respecto a la calidad del servicio y a la dedicación hacia el mismo.

Autores que describen el recorrido en torno a la visibilización de las mujeres en la carrera de medicina $(24,25)$ reportan grandes avances. Sin embargo, vale la pena analizar si ello se debe a políticas de equidad de género claras en los diferentes países o a un cambio en la connotación del ser médico en nuestra sociedad. En la actualidad no se muestran estudios que permitan observar no sólo el acceso sino las posibilidades que tienen las mujeres de mantenerse en los programas de pregrado y de posgrado y tampoco se observa literatura relacionada con su trascurrir en la carrera. Condiciones que motivaron el desarrollo de este estudio.

Por lo anterior el grupo de Salud y Comportamiento planteó un estudio que caracterizó la VBG en una muestra de estudiantes de pregrado y posgrado de la facultad.

\section{Materiales y métodos}

\section{Método}

Exploratorio

\section{Población y muestra}

La población estuvo conformada por estudiantes de pregrado de medicina que se encontraban cursando sus semestres clínicos (V a XII semestres) y estudiantes de posgrado de medicina que se encuentren en proceso de formación. El muestreo fue intencionado y consistió en las y los estudiantes que por semestre voluntariamente accedieran a responder el cuestionario, fueron incluidos en el estudio. Participó un total de 201 estudiantes, 170 de pregrado y 31 de posgrados (medicina interna, cirugía general y ginecología). La mayoría de las/los estudiantes eran mujeres (62\%).

\section{Técnicas y procedimientos}

Posterior a la prueba piloto realizada con estudiantes de la UMNG que se encontraban rotando por Salud Pública, se procedió a realizar la aplicación de los cuestionarios en la población a estudio, en el periodo comprendido entre 
septiembre de 2012 y febrero de 2013. Se concertaron los permisos y espacios con los coordinadores de semestre, así como con los jefes de servicio en el caso de los estudiantes de posgrado.

\section{Instrumentos y medidas}

Se diseñó un cuestionario de conocimientos y actitudes específico para las condiciones de la Violencia basada en el Género en la facultad de medicina teniendo como referencia los estudios realizados por Nagata-Kobayashi et al.(26); la Federación Internacional de Planificación Familiar, Región del Hemisferio Occidental (IPPF-RHO) para la detección sistemática de VBG (27) y la Encuesta Nacional sobre Violencia contra las Mujeres realizada en México en el año 2003 (28). El cuestionario fue diseñado en dos segmentos. El primer segmento identificó claramente el tipo de VBG a la cual él o la estudiante percibieron haber estado expuestos, con una escala tipo Likert se identificó la frecuencia de su ocurrencia, la persona (compañero/a, profesor/a, enfermero/a, residente, paciente) que generó la situación de violencia y el semestre o año en el que se presentó. Y un segundo segmento que hizo referencia a la respuesta (emocional y recursos) personal secundaria al suceso.

\section{Manejo de los Datos}

Se utilizó el módulo Teleform para la captura de los datos y su análisis se realizó a través de SPSS 21.

\section{Aspectos éticos}

Se obtuvo la aprobación del Comité de Ética de la Universidad. Se diligenciaron los consentimientos informados donde se explicaron los aspectos relacionados con confidencialidad y participación voluntaria tanto a la Facultad de Medicina como a los y las estudiantes que participaron. No existieron nombres o códigos de identificación en los cuestionarios (excepto para conocer si era pregrado, posgrado); tampoco se solicitaron nombres de docentes, compañeros o autoridades de la institución. Los datos se utilizaron para fines científicos, fueron conocidos únicamente por los investigadores y se publicaron sin hacer mención específica a personas o lugares de rotación.

\section{Resultados}

La muestra intencionada consistió en un total de 201 estudiantes, 170 de pregrado y 31 de posgrados (medicina interna, cirugía general y ginecología), el 62\% fueron de sexo femenino, lo cual pudo deberse a que la proporción de mujeres en la facultad de medicina es mayor, comportamiento similar al observado a nivel nacional (Teniendo en cuenta el informe de la División de Admisiones y Registro de la Facultad de Medicina de la Universidad Militar Nueva Granada para el primer semestre de 2013 y las estadísticas provenientes del SNIES y SPADIES) para el mismo periodo. Los estudiantes de posgrado que accedieron a responder el cuestionario, se encontraban en primer y tercer año de formación, lo anterior pudo deberse a que los residentes de segundo y cuarto año generalmente se encuentran realizando su rotación por fuera del Hospital de base y no se estableció contacto con los participantes en otras plazas.

\section{Causa de la Violencia}

Se analizó la violencia basada en el género como aquella producida por el hecho de ser hombre o mujer, tener una creencia religiosa ó raza y/o tener una orientación sexual no heterosexual. El 94\% de los/las estudiantes percibieron este tipo de violencia asociada con el hecho de ser mujer, seguido por la orientación no heterosexual en un $8.5 \%$, las creencias religiosas (5.1\%) y la raza (3.4\%). (Figura 1)

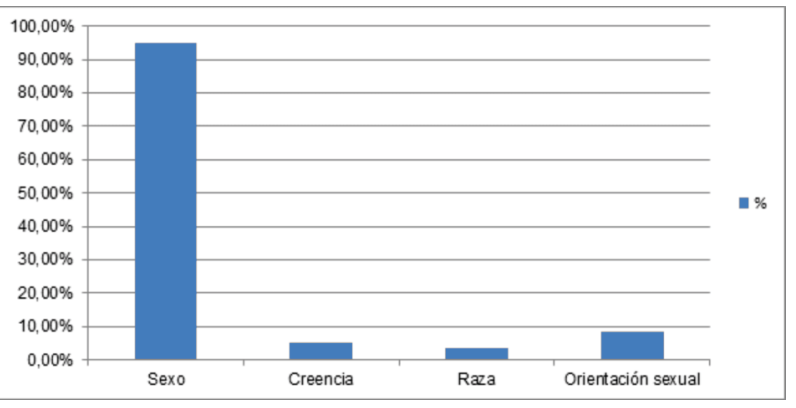

Figura 1. Tipo de VBG en los y las estudiantes de medicina de pre y posgrado de la UMNG 2012-2013

Este tipo de acercamiento hacia la VBG nos permitió observar que, a pesar de tener una aproximación más amplia en esta investigación, en la realidad un gran componente de la violencia basada en el género en la facultad de medicina correspondió a violencia contra las mujeres, seguida por la producida por tener una orientación sexual no heterosexual.

\section{Tipo de Violencia (Psicológica-Física-Sexual)}

El tipo de violencia que fue reportada más frecuentemente por los y las estudiantes es la Violencia psicológica. El $65.7 \%$ de los/las estudiantes refirieron haber recibido un trato hostil durante su proceso formativo, el 31\% manifestó que se han referido hacia ellas/os de manera despectiva o con apodos y el $31 \%$ reportó haber sido gritado. Uno de los comentarios que recibieron los/las estudiantes es que se 
duda acerca de su futuro como profesional en un $42 \%$. Al 21\% le asignaron actividades adicionales como castigo y que no se encuentran dentro del reglamento estudiantil como los turnos. Entre el 17 y $20 \%$ de los/las estudiantes se les negó realizar algunos procedimientos o se les sugirió realizar otro tipo de especialización por el hecho de ser mujer. El 39\% de los estudiantes percibió que le han asignado tareas específicas y diferenciales por ser hombre o por ser mujer.(Figuras 2 y 3 )

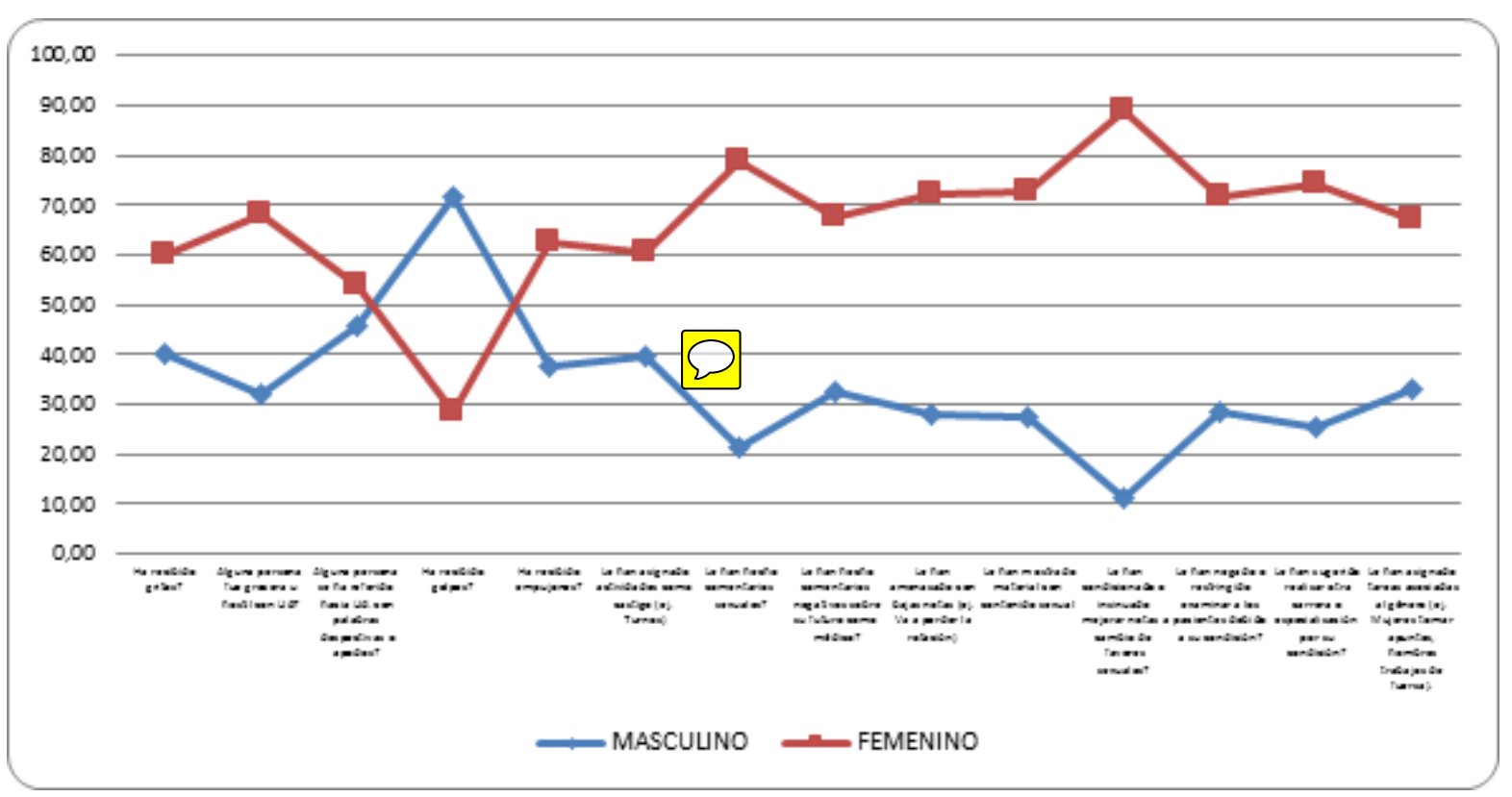

Figura 2. Manifestaciones de violencias en las y los estudiantes de la facultad de medicina UMNG 2012-2013.

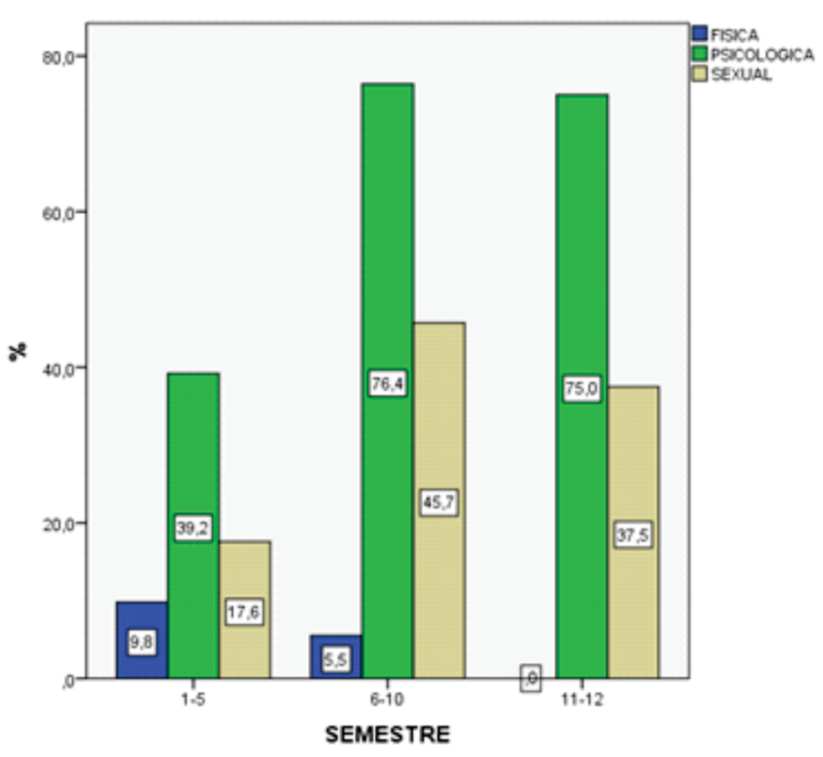

Figura 3. VBG por nivel de formación en estudiantes de pregrado y posgrados de la facultad de medicina de la UMNG 2012-2013.

La Violencia física fue una condición que se analizó en el cuestionario para tener una idea completa de las diferentes formas en las cuales se presenta la violencia basada en el género en el contexto educativo. Los/las estudiantes manifestaron en un $6.5 \%$ haber recibido golpes y empujones por su condición, este fue el único ítem en el cual los hombres reportaron más que las mujeres.

La Violencia Sexual fue reportada en el contexto de la facultad de medicina. El 35.3\% de los estudiantes que participaron en el estudio les han hecho comentarios sexuales, al 5.5\% le han mostrado material con contenido sexual y al $4.5 \%$ se les ha sugerido subir las notas a cambio de favores sexuales. De las personas que reportaron estas situaciones, sólo un hombre realizó este reporte, el resto de la población correspondió a mujeres $(\mathrm{x}=74)$.

\section{Quien ejerce la VBG}

Cuando se hace referencia a la persona que está ejerciendo la violencia, se observó que las personas que mayormente lo hacen son los profesores, seguidos por los estudiantes que se están formando en una especialidad. En un menor porcentaje estudiantes de semestres superiores, personal de apoyo y pacientes. (Figura 4.). 


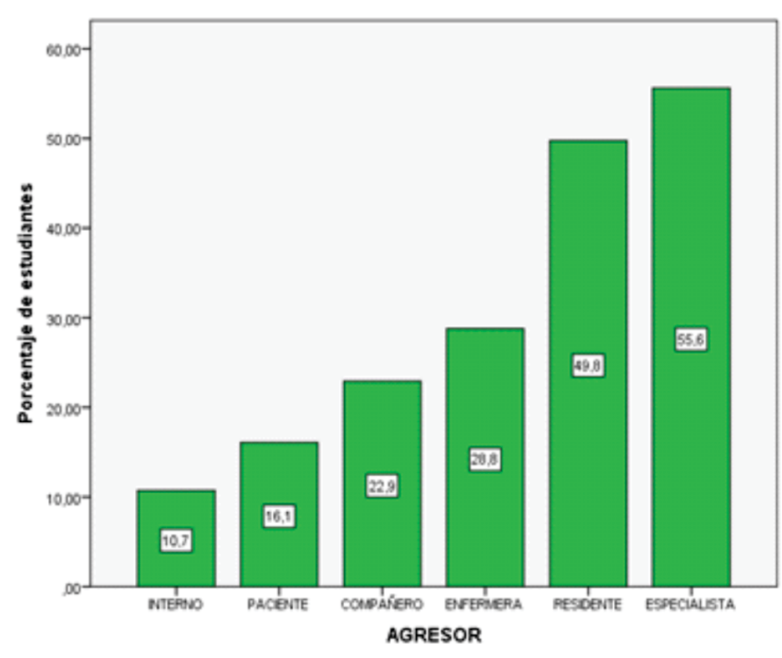

Figura 4. Persona quien ejerce la VBG en estudiantes de pregrado y posgrados de la facultad de medicina de la UMNG 2012-2013

\section{Respuesta (emocional y recursos)}

En cuanto a las respuestas que los/las estudiantes tuvieron con relación a las situaciones presentadas, se observó que la respuesta más frecuente tanto para hombres como para mujeres fue ignorar la situación (41\%), seguida de contarle a un amigo (26.8\%). Esta última más frecuente en mujeres que en hombres. (Tabla 1).

Tabla 1. Respuestas utilizadas por las y los estudiantes de la UMNG con ocasión de la VBG 2012-2013.

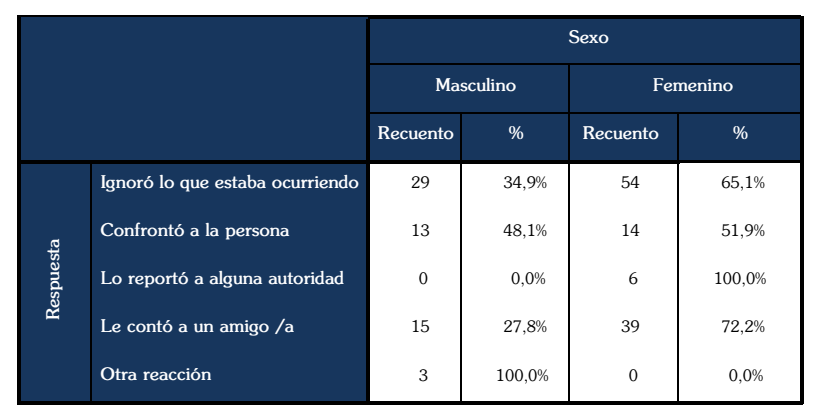

Permite observar la respuesta de los estudiantes ante una situación de violencia. Puede observarse que hacer negación de lo ocurrido es una de las más frecuentes, seguido por contarle a un amigo. No se presentó un solo caso en hombres donde se reportara del hecho a la Universidad.

Las consecuencias identificadas por los y las estudiantes derivadas de la situación presentada fueron en primer lugar, el ignorar lo sucedido y actuar como si este hecho no se hubiera presentado, seguida por la decisión de aumentar su esfuerzo en la materia en mención y en tercer lugar algunos estudiantes optaron por no volver a participar en la actividad académica donde se generó la situación. (Tabla 2).

Tabla 2. Consecuencias percibidas por las y los estudiantes de la UMNG con ocasión de la VBG 2012-2013.

\begin{tabular}{|c|c|c|c|c|c|}
\hline & \multirow{3}{*}{ Tipo de consecuencias } & \multicolumn{4}{|c|}{ Sexo } \\
\hline & & \multicolumn{2}{|c|}{ Masculino } & \multicolumn{2}{|c|}{ Femenino } \\
\hline & & Recuento & $\%$ & Recuento & $\%$ \\
\hline \multirow{6}{*}{ 壾 } & Dejó de asistir a la actividad & 0 & $0,0 \%$ & 1 & $100,0 \%$ \\
\hline & No volvió a participar & 1 & $9,1 \%$ & 10 & $90,9 \%$ \\
\hline & Pensó en retirarse & 6 & $42,9 \%$ & 8 & $57,1 \%$ \\
\hline & Ignoró lo que estaba ocurriendo & 31 & $33,7 \%$ & 61 & $66,3 \%$ \\
\hline & Se esforzó más & 17 & $40,5 \%$ & 25 & $59,5 \%$ \\
\hline & Otra reacción & 1 & $25,0 \%$ & 3 & $75,0 \%$ \\
\hline
\end{tabular}

Como consecuencia de la situación de violencia, los/as estudiantes reportaron de igual manera que hacer caso omiso y esforzarse más en las actividades propias del servicio fueron las más frecuentes. Una estudiante reportó haberse retirado debido a la situación presentada.

En general, las características de la violencia como su tipología, el nivel de formación y la persona que la ejerce son consistentes, es decir, se presentó más frecuentemente la violencia psicológica, en semestres donde ya se encuentran rotando por los Hospitales y quien más frecuentemente ejerció la violencia fue el profesional especialista.

\section{Discusión}

¿Podría hablarse de formas de violencias sutiles, poco perceptibles, tan agudas que la sociedad pierda la habilidad de reconocerlas ó que pudieran confundirse con estilos de enseñanza aprendizaje? ¿En qué momento las limitaciones socioculturales validan un comportamiento en un contexto específico? El propósito de este estudio fue identificar si estas formas "leves" de violencia se estaban dando en el contexto de la enseñanza-aprendizaje en medicina y como las personas respondieron a estas situaciones en su cotidianidad.

Lo que pudo evidenciarse es que la Violencia basada en el Género en la facultad de medicina de la Universidad Militar Nueva Granada, se presentó como una problemática presente y que distó de ser un hecho delicado, leve o sutil. El 94\% de las personas que dieron respuesta al cuestionario y que refirieron haber percibido Violencia basada en el Género, se debió a su condición de ser mujer. Lo que reafirma los planteamientos de la CEDAW(4) y evidencia la importancia de analizar las condiciones de la violencia contra la mujer en los ámbitos no sólo privados sino públicos(9).

El comportamiento de la VBG en la población estudiada, 
tuvo una distribución similar a la encontrada en los estudios desarrollados por la Universidad de Tokio(21), la Universidad Mac Master (19) y los realizados por la Asociación Médica Americana(22). Tanto para las universidades en mención como para el contexto del presente estudio, la VBG fue una condición frecuente y con hallazgos inesperados como la presencia de algún tipo de violencia física. De allí que sea necesario profundizar por qué en un entorno de salud - donde se manejan conceptos como bienestar, equidad, diversidad, respeto a los pacientes - se podría estar manejando un lenguaje paralelo y contradictorio.

Estas conductas se presentaron en la totalidad de los niveles de formación, sin embargo con mayor frecuencia, en periodos donde se presentó un cambio. Por ejemplo, los semestres donde los y las estudiantes ingresaron a sus prácticas clínicas y el primer año de residencia en los posgrados (20). De allí la necesidad de realizar un proceso de sensibilización previa al ingreso de los/las estudiantes y los profesionales de salud y docentes en dichos momentos de formación. De igual manera, reforzar los procesos de actualización y educación en docencia universitaria en hospitales. En el caso de los docentes, se han realizado estudios acerca de "la angustia docente" en el ámbito escolar(29), sería interesante llevar el estudio de esta temática al ámbito universitario para identificar en este contexto al docente como potencial víctima o victimario.

La violencia psicológica fue el tipo de violencia utilizado más frecuentemente en el ámbito educativo de la facultad de medicina, lo que podría estar asociado a una restricción sociocultural que podría ser identificada a través de frases coloquiales como "la letra con sangre entra", que según Kalbermatter (30), metaforiza no solo el aprender como doloroso, sino la existencia de pasiones en este proceso. De esta manera en algunos contextos la exigencia académica y éxito, podrían verse erróneamente asociados con técnicas restrictivas del aprendizaje. Estas prácticas podrían ser validadas por los y las estudiantes como la norma social, lo que podría explicar por qué una de las consecuencias más enunciadas por los estudiantes, fue que la situación los llevó a esforzarse más en su cotidianidad. Lo anterior podría llevar a un círculo vicioso de reconocimiento y validación que puede generar una gran limitante para la formulación de estrategias de cambio a futuro.

En la actualidad, a pesar de tener la posibilidad de ingresar a la carrera de medicina; en el entorno donde se lleva a cabo la docencia podrían persistir factores sociales, culturales y relacionales (15) tales como las normas que otorgan a los hombres el control, la aceptación del uso de la violencia para resolver conflictos y los papeles rígidos para cada sexo. Lo que explicaría la alta frecuencia de violencia sexual (acoso sexual) en nuestro contexto y en la literatura de referencia en los ámbitos universitarios $(16,31,32)$ y podría estar visibilizando un el ámbito académico como un escenario en el cual se validan las conductas violentas, lo que se encuentra acorde con los postulados de Newman (22).

La VBG por el hecho de tener una orientación no heterosexual fue la segunda causa de violencia en la población estudiada, situación que deberá ser analizada en estudios posteriores a mayor profundidad dado que estudios recientes realizados en países como Chile, han demostrado que este tipo de violencia se presenta en las instituciones educativas y que aportan casi el 10\% de la deserción (33).

Los y las estudiantes de la facultad de medicina no comentan la situación vivida como pudo observarse en el presente estudio, condición que había sido identificada previamente en Chile, en donde entre el 25-50\% de los/las estudiantes no reportaron una situación de violencia sexual en el último año porque no sabían que ello era un crimen o algo serio (34). Lo que hizo reflexionar que desde el punto de vista institucional, no se cuenta en la actualidad con un mecanismo claro de reporte de estas circunstancias, diferente a los procesos de asesoría psicológica.

A manera de conclusión, el primer paso para erradicar el uso de la violencia en los espacios de aprendizaje es su reconocimiento, seguido de la sensibilización de la población y el planteamiento de políticas institucionales diseñadas a través de estrategias participativas. Este estudio permitió el reconocimiento de la problemática y orientó nuevas perspectivas para profundizar acerca de la temática como analizar las condiciones de la docencia misma, así como el contexto en el cual se lleva a cabo y el papel de los diferentes actores del proceso de enseñanza-aprendizaje.

Desde lo institucional, se recomienda establecer una política de sensibilización, detección temprana y establecimiento de correctivos para que la comunidad educativa encuentre un espacio de confianza, y la certeza que su caso será escuchado y que su derecho a una vida libre de violencia será restablecido.

\section{Conflicto de intereses}

Los autores declaran no tener de manera directa o indirecta, ningún tipo de conflicto de intereses financieros, académicos o laborales que puedan poner en peligro la validez de este estudio.

\section{Financiación}

Este trabajo se realizó con el apoyo financiero de la Universidad Militar Nueva Granada. 


\section{Referencias}

1. Sánchez M, Enciso M, Fernández E. Marco Conceptual. In: Procuraduría General de la Nación, UNFPA, editors. Vigilancia superior a la garantía de los derechos desde una perspectiva de género, con énfasis en mujeres y adolescentes Guía pedagógica y operativa para el seguimiento y la vigilancia. 2nd ed. Bogota, Colombia: TC Impresiones; 2006. p. 21-45.

2. Garcia-Moreno C, Heise L, Jansen HAFM, Ellsberg M, Watts C. Violence against Women. Science (80). 2005;310(5752):1282-3.

3. Salamea L, Bueso M, Patriota T, Pisani M, Ruiz E, Lopez C, et al. Estudio sobre tolerancia social e institucional a la violencia basada en género en Colombia. [Internet]. Bogota, Colombia; 2010. Available from: http://www.mdgfund.org/ sites/default/files/GEN_ESTUDIO_Colombia_Tolerancia social e institucional a la violencia de género.pdf

4. Organización de las Naciones Unidas. Convención sobre la eliminación de todas las formas de discriminación contra la mujer. 1994 [cited 2014 May 14];1-16. Available from: http://www.un.org/womenwatch/daw/cedaw/text/sconven tion.htm

5. Hernández Cordoba A, Bruno Nn, Rivera L. Violencia conyugal como oportunidad de reencuentro y de reconstrucción existencial. Bogotá; 1999.

6. Jenkins A. Invitations to Responsibility: The therapeutic engagement of men who are violent and abusive. Adelaide: Dulwich Centre Publications; 1990. 229-242 p.

7. Amnistia Internacional. Informe 2013 Amnistía Internacional, el estado de los derechos humanos en el mundo [Internet] EditorialAmnistía, Internacional(EDAI), editors. Madrid: ArtesGráficasENCO Madrid(España); 2013. 91 p. Available from: http://files.amnesty.org/air13/ AmnestyInternational_AnnualReport2013_complete_es.pdf

8. Instituto Nacional de Medicina Legal y Ciencias Forenses. Forensis mujeres datos para la vida [Internet]. Bogota, Colombia; 2010. Available from: http:// www.medicinalegal.gov.co/masatugo-2004-2008

9. Vélez A, Espriella A de la. Aproximación a la salud sexual y reproductiva de las mujeres desplazadas en Colombia. Profamilia [Internet]. 2004 [cited 2012 Nov 1];74. Available from : ht t p : / s c holar.google.com/ scholar?hl=en\&btnG=Search\&q=intitle:Aproximación $+\mathrm{a}+\mathrm{la}+$ Salud+Sexual+y+Reproductiva+de+las+mujeres+desplazadas + en+Colombia\#1

10. Profamilia. Encuesta Nacional de Demografía y Salud [Internet]. Bogota, Colombia: Profamilia; 2011. 361-399 p. Available from: http://www.profamilia.org.co/ encuestas/Profamilia/Profamilia/index.php?option=com_co ntent\&view $=$ article\&id $=63 \&$ Itemid $=7$

11. Paredes O, Sanabria-Ferrand P, González-Quevedo L, Moreno-Realphe S. "Bullying" en las facultades de medicina colombianas, mito o realidad. Rev Med. 2010;18(49):161-72.

12. Moreno-Cubillos C, Osorio-Gómez L, Sepúlveda-Gallego LE. Violencia sexual contra las estudiantes de la Universidad de Caldas. Estudio de corte transversal. Rev colomb Obs ginecol. 2007;58(2):116-23.
13. Secretaría de Seguridad Pública. Manual Prevención de la Violencia de Género en Diversos Contextos. Mexico: Gobierno Federal de México; 2012. p. 1-45.

14. Organización Panamericana de la Salud. Repercusión de la violencia en la salud de las poblaciones americanas. In: 44 Consejo Directivo de la Organización Panamericana de la Salud. Washington, D.C.: PAHO; 2003. p. 2-19.

15. Heise L 1. Violence against women: an integrated, ecological framework. Violence Against Women. 1998;4(3):262-90.

16. Moreno C, Sepúlveda L, Restrepo L. Discriminación y violencia de género en la Universidad de Caldas. Hacia la Promoción la Salud [Internet]. 2012;17(1):59-76. Available from: http://promocionsalud.ucaldas.edu.co/ downloads/Revista17(1)_4.pdf

17. Morgan JF, Porter S. Sexual harassment of psychiatric trainees: experiences and attitudes. Postgrad Med J [Internet] 1999 Jul;75(885):410-3. Available from: http:// www.pubmedcentral.nih.gov/articlerender.fcgi?artid=17412 98\&tool=pmcentrez\&rendertype $=$ abstract

18. Gadomski a M, Wolff D, Tripp M, Lewis C, Short LM. Changes in health care providers' knowledge, attitudes, beliefs, and behaviors regarding domestic violence, following a multifaceted intervention. Acad Med [Internet]. 2001 Oct; $76(10): 1045-52$. Available from: http://www.ncbi.nlm.nih.gov/pubmed/11597847

19. Margittai KJ, Moscarello R, Rossi MF. Forensic aspects of medical student abuse: a Canadian perspective. Bull Am Acad Psychiatry Law [Internet]:1996 Jan;24(3):377-85. Available from: http://www.ncbi.nlm.nih.gov/pubmed/ 8889136

20. Dikmeta E, Top M, Ergin G. An examination of mobbing and burnout of residents. Turkish $\mathrm{J}$ psychiatry [Internet]. 2011 $\mathrm{J}$ a n ; 2 2(3): $137-49$. Ava i lable from http://www.ncbi.nlm.nih.gov/pubmed/21870303

21. Nagata-Kobayashi S, Sekimoto M, Koyama H, Yamamoto W, Goto E, Fukushima O, et al. Medical student abuse during clinical clerkships in Japan. J Gen Intern Med [Internet]. 2006 Mar [cited 2013 May 27];21(3):212-8. Available from: http://www.pubmedcentral.nih.gov/articlerender.fcgi?artid= 1828085\&tool=pmcentrez\&rendertype $=$ abstract

22. Rappley M. Section on Medical Schools. In: Optimizing the Learning Environment: Exploring the Issue of Medical Student Mistreatment [Internet]. Joint Education Program with the AMA Medical Student Section and AMA the Council on Medical Education; 2011. p. 2-14. Available from:http://www.ama-assn.org/resources/ doc/medicalschools/sms-a11-mistreatment

23. Newman L. James Mackenzie Lecture 1991. Second among equals. Br J Gen Pract [Internet]. 1992 Mar;42(355):71-4. Available from: http://www.pubmedcentral.nih.gov/ articlerender.fcgi?artid=1371928\&tool=pmcentrez\&renderty pe $=$ abstract

24. Scott JM. Women and the GMC : the struggle for representation. JR Soc Med. 1988;81(March):164-6.

25. Risberg G, Johansson EE, Westman G, Hamberg K. Gender in medicine - An issue for women only ? A survey of physician teachers ' gender attitudes. Int J Equity Health. 2003;8:1-8. 
26. Arimatsu M, Wada K, Yoshikawa T, Oda S, Taniguchi H, Aizawa Y, et al. An epidemiological study of work-related violence experienced by physicians who graduated from a medical school in Japan. J Occup Health [Internet]. 2008 Jan;50(4):357-61. Available from: http://www.ncbi.nlm. nih.gov/pubmed/18560204

27. Finn T. A Guide for Monitoring and Evaluating PopulationHealth-Environment Programs. MEASURE Evaluation, USAID; 2007. 3-165 p.

28. Olaiz G, Rico B, Del Río A. Encuesta Nacional sobre Violencia contra las Mujeres INSP. 1st ed. Cuernavaca, Morelos; 2003.

29. León E. Angustia docente: una revisión de la investigación del malestar y la violencia. Rev Innovar. 2010;91-110.

30. Kalbermatter MC. La violencia: un fenómeno social que atraviesa la escuela. In: Violencia ¿esencia o construcción? ¿víctimas o victimarios? 1st ed. Córdoba: Editorial Brujas; 2006. p. 83-111.

31. Anderson C. Prevention and Intervention of Sexual Violence in Schools: Talking about It. 2000. 1-27 p.

32. Caballero MC. El acoso sexual en el medio laboral y académico. In: Viveros M, Góngora A, Martínez M, editors. Saberes, culturas y derechos sexuales en Colombia. 1st ed. Bogotá: Editorial Tercer Mundo del gupo TM S.A.; 2006. p. 429-50.

33. Barrientos-Delgado J, Cardenas-Castro M, Gómez-Ojeda F. Características sociodemográficas, bienestar subjetivo y homofobia en una muestra de hombres gay en tres ciudades chilenas Socio-demographic characteristics, subjective wellbeing, and homophobia experienced by a sample of gay men from three cities in. Cad Saúde Pública, Rio Janeiro. 2014;30(6):1259-69.

34. Lehrer JA, Lehrer EL, Oyarzún PB. Violencia sexual en hombres y mujeres jóvenes en Chile: Resultados de una encuesta a estudiantes universitarios. Rev Med Chile. 2009; 137:599-608 